\title{
N-Doped Carbon Dots for Visual Recognition of 4-Nitroaniline and Use in Fluorescent Inks
}

\author{
Nilanjana Nandi, ${ }^{a}$ Priyanka Sarkar, ${ }^{a}$ Kalyanasis Sahu ${ }^{* a}$ \\ a Department of Chemistry, Indian Institute of Technology Guwahati, Guwahati 781039, \\ Assam, India.*E-mail: ksahu@iitg.ac.in (K.S.).
}

\section{Materials}

5-aminoisopthalic acid, 3,5-diaminobenzoic acid, and other analytes were purchased from Sigma-Aldrich. (Shanghai, China) while absolute ethanol and other metal salts were from Merck. All reagents and solvents were used as received without further purification. Milli-Q deionized water (18.2 M $\Omega$ ) was used for preparing aqueous solutions.

\section{Synthesis Procedure}

NCD-1 and NCD-2 were synthesized from 5-aminoisopthalic acid and 3,5-diaminobenzoic acid. One of the precursors was dissolved in $15 \mathrm{ml}$ ethanol and sonicated for $1 \mathrm{~h}$, and stirred for $30 \mathrm{~min}$ to form a homogeneous mixture. The solution was then transferred into a Teflon-lined autoclave inner vessel and placed in an oven at $160{ }^{\circ} \mathrm{C}$ for 12 hours. All resultant NCDs solution was cooled down to room temperature and filtered with a $0.22 \mu \mathrm{m}$ PTFE membrane to remove any solid byproducts. Then, the NCD solution was placed in a dialysis tube (MWCO $1000 \mathrm{Da}$ ) for 3 days. Finally, the purified solid product was obtained after drying the solution at $50{ }^{\circ} \mathrm{C}$.

\section{Characterization}

UV-visible absorption spectra and steady-state fluorescence were recorded in a Perkin-Elmer lamda750 spectrophotometer and Jobin-Yvon FluoroMax 4 by using a $1 \mathrm{~cm}$ path length cuvette. Fluorescence decays were collected in a time-correlated single-photon counting (TCSPC) set up from Horiba instruments using a $375 \mathrm{~nm}$ laser diode. The images of the nanomaterials were taken through carried out in JEOL JEM $2100 \mathrm{~F}$ transmission electron microscope (TEM) operating at $200 \mathrm{kV}$. Image-J software was used to analyze the size distribution of the nanomaterials, considering the sizes of at least 50 particles. The structure was identified by Xray diffraction analysis (Rigaku SmartLab $9 \mathrm{~kW})$ using $\mathrm{Cu} \mathrm{K \alpha}(\lambda=1.54 \AA$ ) as the source with 
a 9-kW power. The elemental composition was determined by X-ray photoelectron spectroscopy (PHI 5000 VersaProbe III). UV treatment analysis was carried out by using a Spectrolite UV lamp. Raman scattering measurements were performed in a Horiba Raman spectrometer (LabRam HR800, Jobin Yvon) with a laser excitation of $488 \mathrm{~nm}$. Fourier transform infrared (FTIR) spectra of all samples were recorded in a Perkin Elmer instrument in attenuated total reflectance (ATR) mode. DFT calculation was measured by using Gaussian 09 program B3LYP/6-31G $+(\mathrm{d}, \mathrm{p})$.

\section{Quantum yield (QY) measurement}

The relative QY of the NCD-1 and NCD-2 were estimated by considering quinine sulphate $\left(\mathrm{QY}_{\mathrm{R}}=54.4 \%\right.$ in $\left.0.1 \mathrm{M} \mathrm{H}_{2} \mathrm{SO}_{4}\right)$ as a standard. The QY of a sample was calculated according to the following equation:

$$
\mathrm{QY}(\mathrm{NCDs})=\frac{\mathrm{I}}{\mathrm{I}_{\mathrm{R}}} \times \frac{\mathrm{A}_{\mathrm{R}}}{\mathrm{A}} \times \frac{\eta^{2}}{\eta_{\mathrm{R}}^{2}} \times \mathrm{QY}_{\mathrm{R}}
$$

Where QY is the measuring quantum yield of NCDs. I and $I_{R}$ are integrated emission intensities of NCD and quinine sulfate, respectively. $\eta$ is the refractive index (1.33 for water), and A is the optical density. The absorbance was always kept below 0.1 at the excitation wavelength.

\section{Cyclic Voltammetry (CV) Studies}

A three-electrode cell was used for $\mathrm{CV}$ measurements of $\mathrm{NCDs}$, where saturated $\mathrm{Ag} / \mathrm{AgNO} \mathrm{N}_{3}$, platinum wire, and glassy carbon eletrode serve as reference, counter, and working electrode, respectively. $\mathrm{CV}$ studies were done at room temperature and in argon environment. Tertabutylammoniumhexafluorophosphate $(0.1 \mathrm{M})\left(\mathrm{TBAPF}_{6}\right)$ was utilized as a supporting electrolyte in $\mathrm{CH}_{3} \mathrm{CN}$ and $\mathrm{Fc}^{+} / \mathrm{Fc}$ couple as the reference internally. 

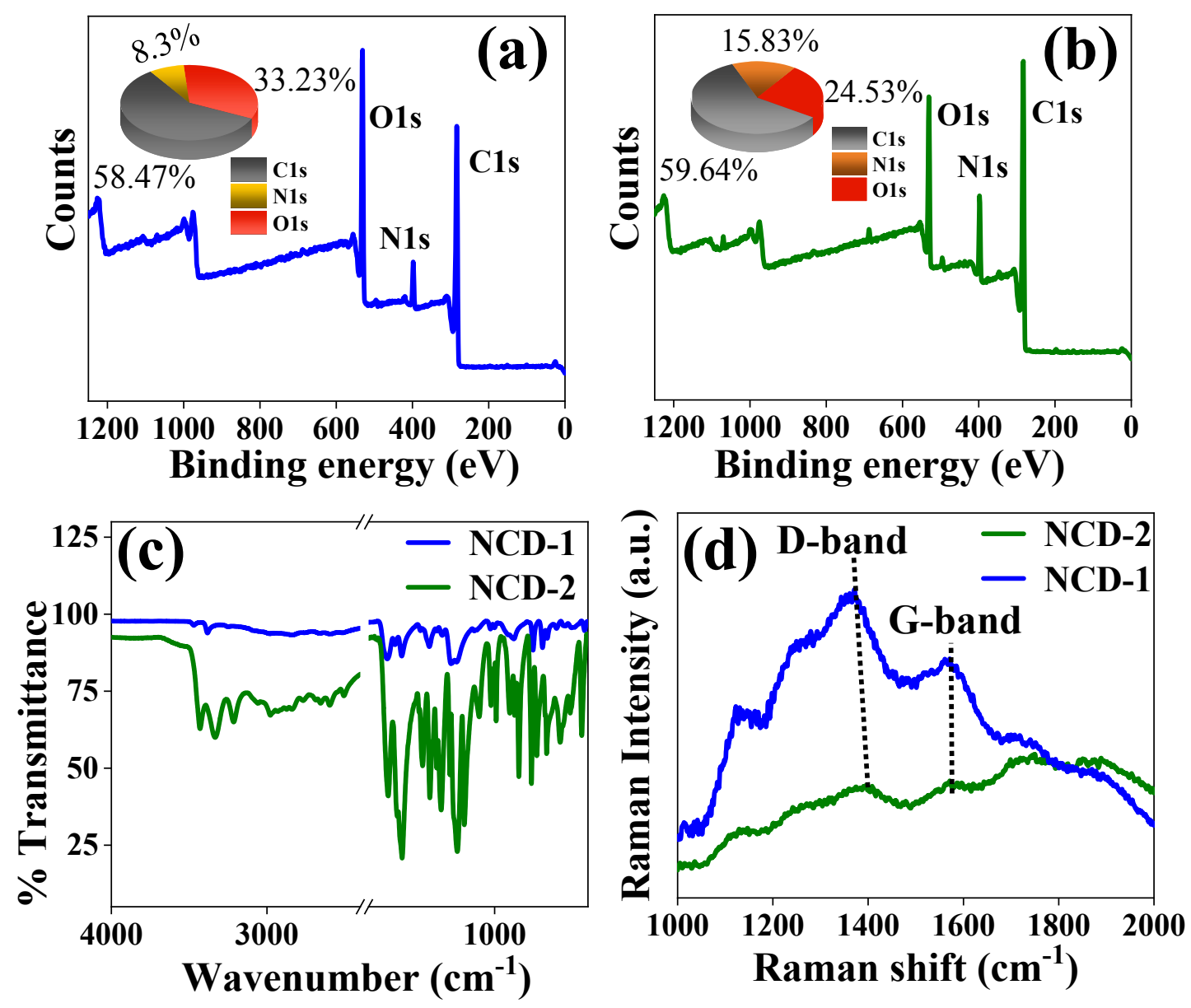

Figure S1. XPS spectra of NCD-1 (a) and NCD-2 (b). (c) FTIR spectra of NCD-1 ana NCD2. (d) Raman Spectra of NCD-1 and NCD-2.

Table S1. Deconvoluted fitting parameter of XPS spectra of NCD-1

\begin{tabular}{|c|c|c|c|c|}
\hline Sample & Spectrum & $\begin{array}{c}\text { Peak position } \\
(\mathrm{eV})\end{array}$ & Peak identity & $\begin{array}{c}\% \text { Of } \\
\text { contribution }\end{array}$ \\
\hline \multirow{11}{*}{ NCD-1 } & \multirow{4}{*}{$\mathrm{C} 1 \mathrm{~s}$} & 284.3 & $\mathrm{C}=\mathrm{C}$ & 30 \\
\hline & & 285.1 & $\mathrm{C}-\mathrm{N}$ & 24 \\
\hline & & 286 & $\mathrm{C}=\mathrm{N}$ & 20 \\
\hline & & 288.8 & $\mathrm{C}=\mathrm{O}$ & 26 \\
\hline & \multirow[b]{3}{*}{$\mathrm{N} 1 \mathrm{~s}$} & 398.9 & Pyridinic-N & 48 \\
\hline & & 399.7 & Amino-N & 39 \\
\hline & & 400.9 & Pyrrolic-N & 13 \\
\hline & \multirow{4}{*}{$\mathrm{O} 1 \mathrm{~s}$} & 531 & $\mathrm{C}-\mathrm{O}$ & 7 \\
\hline & & 532 & $\mathrm{C}-\mathrm{O}-\mathrm{C}$ & 42 \\
\hline & & 533.3 & $\mathrm{C}-\mathrm{OH}$ & 39 \\
\hline & & 534.3 & $\mathrm{O}=\mathrm{C}-\mathrm{O}$ & 12 \\
\hline
\end{tabular}


Table S2. Deconvoluted fitting parameter of XPS spectra of NCD-2

\begin{tabular}{|c|c|c|c|c|}
\hline Sample & Spectrum & $\begin{array}{c}\text { Peak position } \\
(\mathrm{eV})\end{array}$ & Peak identity & $\begin{array}{c}\% \text { Of } \\
\text { contribution }\end{array}$ \\
\hline \multirow{11}{*}{ NCD-2 } & \multirow{4}{*}{$\mathrm{C} 1 \mathrm{~s}$} & 284.7 & $\mathrm{C}=\mathrm{C}$ & 67 \\
\hline & & 285.5 & $\mathrm{C}-\mathrm{N}$ & 4 \\
\hline & & 286.2 & $\mathrm{C}=\mathrm{N}$ & 13 \\
\hline & & 288.7 & $\mathrm{C}=\mathrm{O}$ & 16 \\
\hline & \multirow[b]{3}{*}{$\mathrm{N}$ 1s } & 398.5 & Pyridinic-N & 19 \\
\hline & & 399.2 & Amino-N & 64 \\
\hline & & 400.2 & Pyrrolic-N & 17 \\
\hline & \multirow{4}{*}{$\mathrm{O} 1 \mathrm{~s}$} & 531 & $\mathrm{C}-\mathrm{O}$ & 9 \\
\hline & & 531.8 & $\mathrm{C}-\mathrm{O}-\mathrm{C}$ & 52 \\
\hline & & 533.3 & $\mathrm{C}-\mathrm{OH}$ & 32 \\
\hline & & 534.1 & $\mathrm{O}=\mathrm{C}-\mathrm{O}$ & 7 \\
\hline
\end{tabular}

Table S3. Quantum yields of NCD 1 and NCD-2 in various solvents.

\begin{tabular}{|l|c|c|}
\hline \multicolumn{1}{|c|}{ Solvent } & NCD-1 & NCD-2 \\
\hline Tetrahydrofuran (THF) & $11.6 \%$ & $8.4 \%$ \\
\hline Acetonitrile (AcCN) & $9.1 \%$ & $6.9 \%$ \\
\hline Ethanol (EtOH) & $12.2 \%$ & $13.4 \%$ \\
\hline DMSO & $20.3 \%$ & $22.7 \%$ \\
\hline Water & $15.6 \%$ & $14.7 \%$ \\
\hline
\end{tabular}



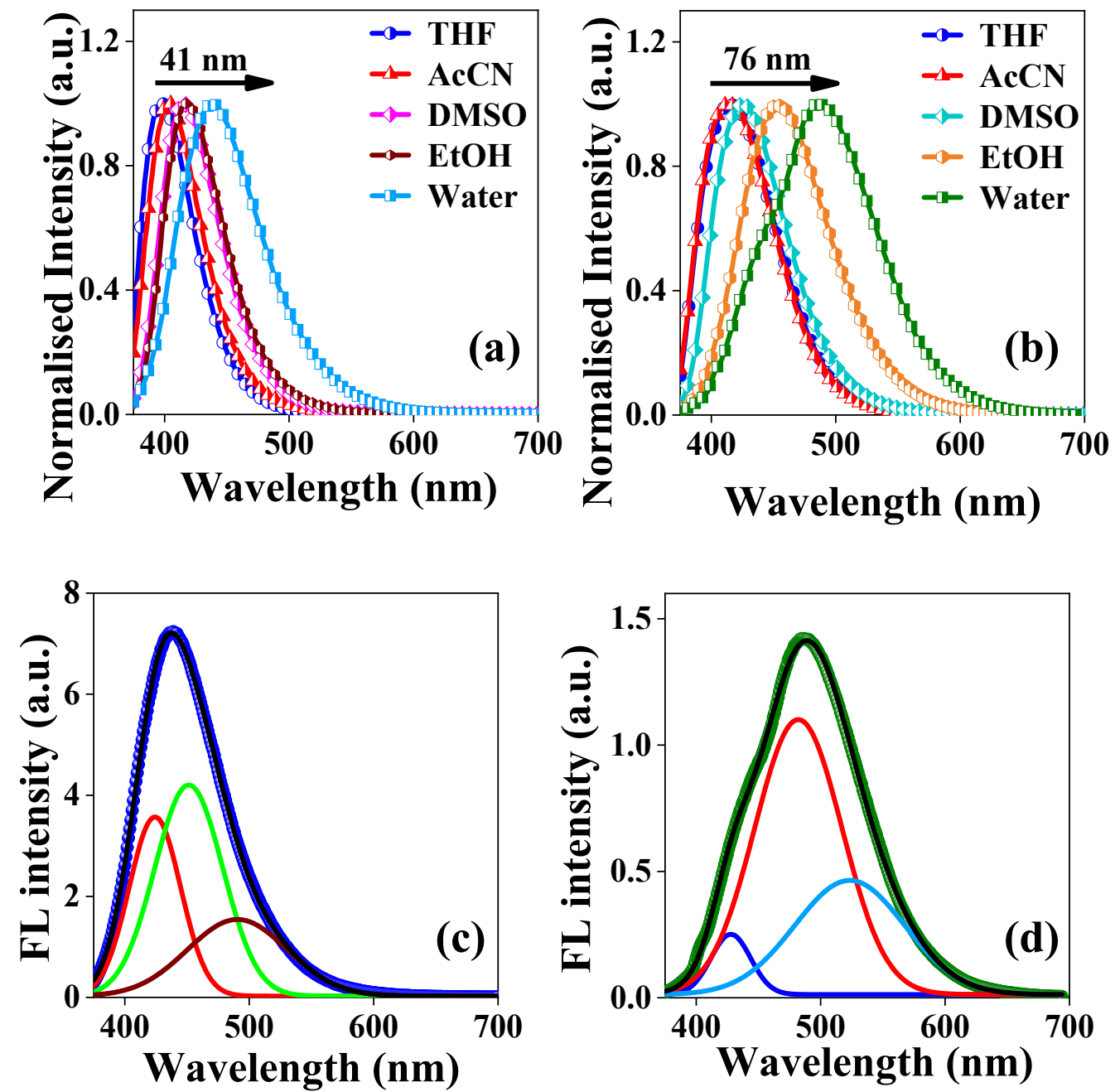

Figure S2. $(\mathrm{a}, \mathrm{b})$ Emission spectra of NCD-1 and NCD-2 in different solvents $\left(\lambda_{\mathrm{ex}}=360 \mathrm{~nm}\right)$; (c, d) decomposition of NCD-1 and NCD-2 emission spectra in water into three different bands using gaussian fitting.
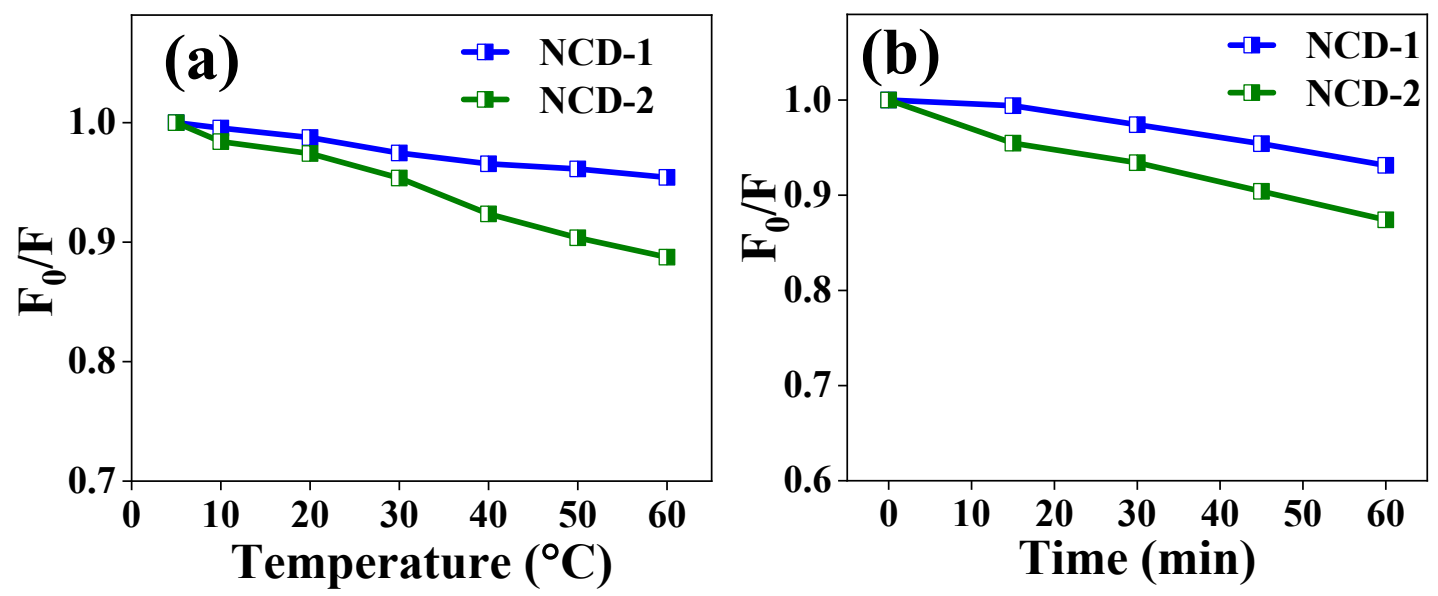

Figure S3. (a) Temperature dependent study of NCD-1 and NCD-2. (b) UV-treatment of NCD-1 and NCD-2. 
Table S4. Solid and solution state decay fitting parameters for NCD-1.

\begin{tabular}{|l|l|l|l|l|l|l|l|l|}
\hline Sample & $\mathbf{A}_{\mathbf{1}}$ & $\mathbf{A}_{\mathbf{2}}$ & $\mathbf{A}_{\mathbf{3}}$ & $\begin{array}{l}\boldsymbol{\tau}_{\mathbf{1}} \\
(\mathbf{n s})\end{array}$ & $\begin{array}{l}\boldsymbol{\tau}_{\mathbf{2}} \\
(\mathbf{n s})\end{array}$ & $\begin{array}{l}\boldsymbol{\tau}_{\mathbf{3}} \\
(\mathbf{n s})\end{array}$ & $\begin{array}{l}\boldsymbol{\tau}_{\text {avg }} \\
(\mathbf{n s})\end{array}$ & $\chi^{\mathbf{2}}$ \\
\hline $\begin{array}{l}\text { NCD-1 } \\
\text { Solution } \\
\text { state }\end{array}$ & 0.31 & 0.32 & 0.37 & 1.88 & 5.51 & 0.33 & 2.47 & 1.14 \\
\hline $\begin{array}{l}\text { NCD-1 } \\
\text { Solid state }\end{array}$ & 0.07 & 0.01 & 0.92 & 1.437 & 14.62 & 0.154 & 0.39 & 1.43 \\
\hline
\end{tabular}

The decays were fitted in a tri-exponential function by using eq 1.

$$
I(t)=A_{1} e^{\left(-t / \tau_{1}\right)}+A_{2} e^{\left(-t / \tau_{2}\right)}+A_{3} e^{\left(-t / \tau_{3}\right)}
$$

Where $\tau_{i} s$ are the decay time of components and $A_{i} s$ are the corresponding contributions.

The average decay time can be further calculated by the eq 2 .

$$
\tau=A_{1} \tau_{1}+A_{2} \tau_{2}+A_{3} \tau_{3}
$$
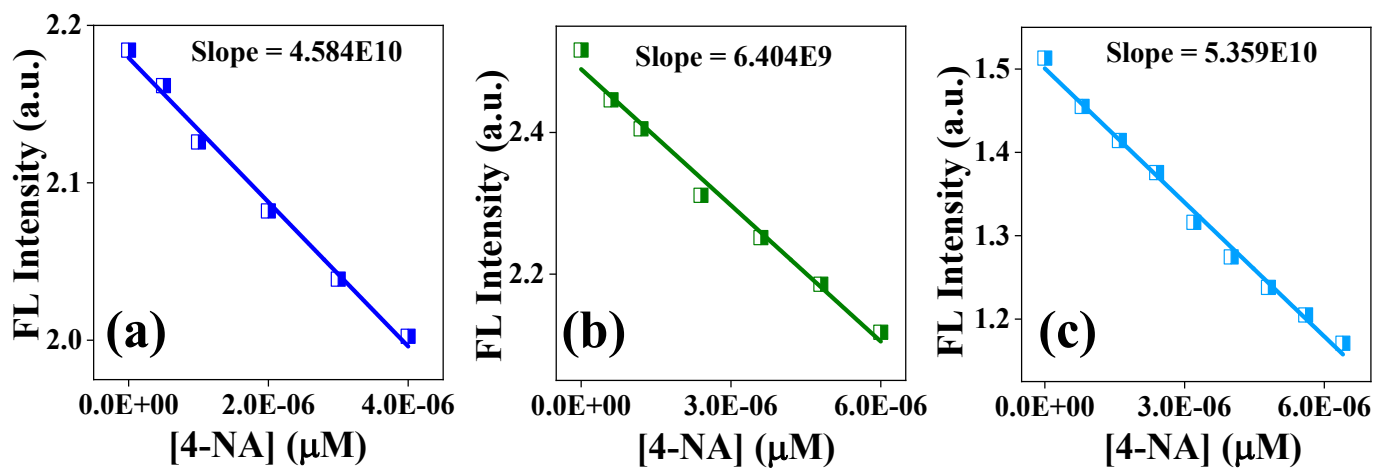

Figure S4. Limit of detection plot of NCD-1 (a), NCD-2 (b) in aqueous medium and (c) NCD-2 in DMSO medium
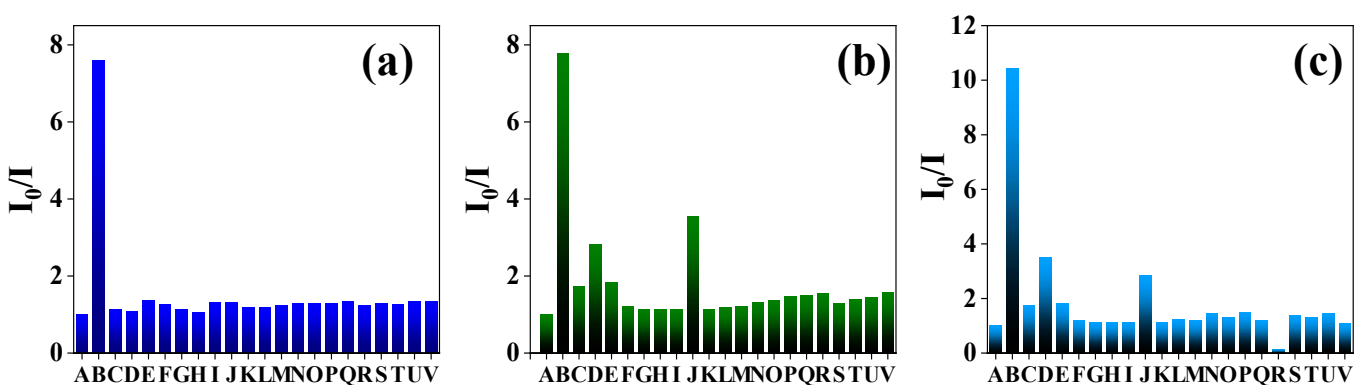

Figure S5. Selectivity study of NCD-1 (a) and NCD-2 (b) in aqueous medium. (c) Selective study of NCD-2 in DMSO medium. Here, A, B, C, D, E, F, G, H, I, J, K, L, M, N, O, P, Q, R, S, T, U, V refers to blank, 4-NA, 1, 3-DNB, 2,4-DNA, 2-NA, 3-NA, 4-bromo-NA, Aniline, $\mathrm{NB}, \mathrm{EDA}, \mathrm{TEA}, \mathrm{Na}^{+}, \mathrm{K}^{+}, \mathrm{Mn}^{2+}, \mathrm{Zn}^{2+}, \mathrm{Cr}^{3+}, \mathrm{Co}^{2+}, \mathrm{CN}^{-}, \mathrm{I}^{-}, \mathrm{Br}^{-}, \mathrm{HCO}_{3}{ }^{-}, \mathrm{CO}_{3}{ }^{2-}$ respectively. 

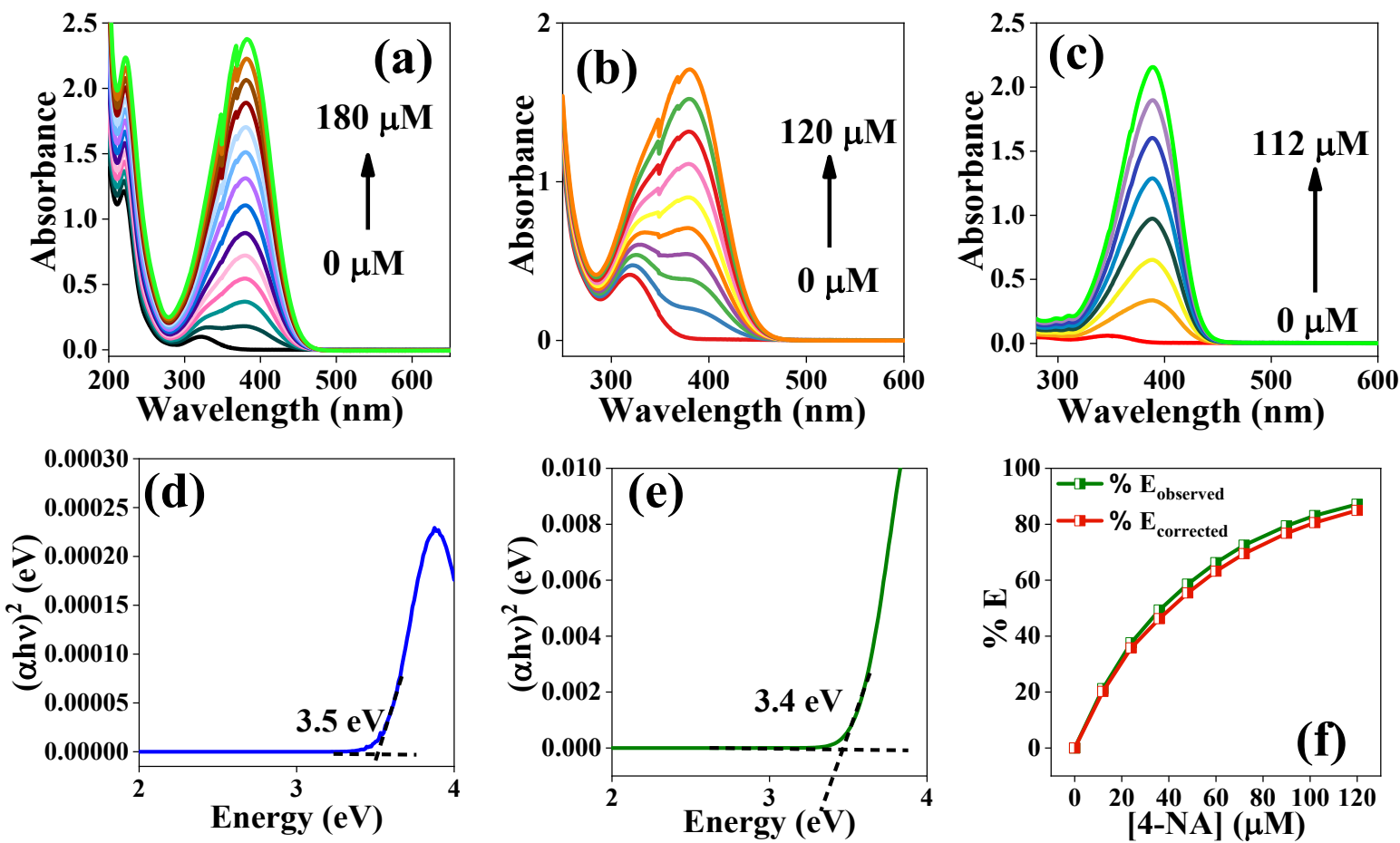

Figure S6. UV-vis absorption spectra after addition of several concentration of 4-NA (a) NCD1, (b) NCD-2 in aqueous solution and (c) NCD-2 DMSO solvent. (d) and (e) UV-onset graph of NCD-1 and NCD-2 respectively, (f) IFE plot of NCD-2 in aqueous medium.

Table S5. Comparison of detection limits for detection of 4-NA using different methods.

\begin{tabular}{|c|c|c|}
\hline Publication & Sensing Methods & Detection limit \\
\hline $\begin{array}{c}\text { Journal of Environmental } \\
\text { Chemical Engineering } 9 \\
\text { (2021) } 105821\end{array}$ & Electrochemical & $0.17 \mu \mathrm{M}$ \\
\hline $\begin{array}{c}\text { Chemosphere } 81 \text { (2010) } \\
430-435\end{array}$ & $\begin{array}{l}\text { High-performance liquid } \\
\text { chromatography }\end{array}$ & $4.5 \mathrm{nM}$ \\
\hline $\begin{array}{c}\text { Talanta } 74(2008) 1400- \\
1407\end{array}$ & UV-photolysis & $1 \mu \mathrm{M}$ \\
\hline $\begin{array}{c}\text { Electroanalysis, } 2007,19, \\
1387-1393\end{array}$ & Glassy carbon electrode & $8 \mathrm{nM}$ \\
\hline $\begin{array}{c}\text { Chromatographia, 2004, 59, } \\
\text { 167-172 }\end{array}$ & $\begin{array}{c}\text { Liquid chromatography } \\
\text { with amperometric } \\
\text { detection. }\end{array}$ & $0.16 \mu \mathrm{M}$ \\
\hline This work & Fluorescence & $\begin{array}{c}111.6 \mathrm{nM} \text { (NCD-1), } \\
68.9 \mathrm{nM} \text { (NCD-2) }\end{array}$ \\
\hline
\end{tabular}


Table S6. Comparison of LOD values by various fluorescence based sensors for the detection of 4-NA.

\begin{tabular}{|c|c|c|}
\hline Publication & Precursor & Detection limit \\
\hline $\begin{array}{c}\text { New J. Chem., 2020, 44, } \\
\text { 10525-10535 }\end{array}$ & $\begin{array}{c}\text { Oyster mushroom-carbon } \\
\text { dots }\end{array}$ & $0.50 \mu \mathrm{M}$ \\
\hline $\begin{array}{c}\text { Analyst, } 2015,140,1428- \\
1431\end{array}$ & W-N-CDs and E-N-CDs & 1 and $0.1 \mu \mathrm{M}$ \\
\hline Material chemistry frontiers & $\begin{array}{l}\text { anthracene based conjugated } \\
\text { triazine framework }\end{array}$ & $1.5 \mu \mathrm{M}$ \\
\hline $\begin{array}{c}\text { Microchimica Acta (2019) } \\
\text { 186: } 457\end{array}$ & $\begin{array}{c}\text { ketone-functionalized } \\
\text { carbazolic porous organic } \\
\text { framework }\end{array}$ & $1.1 \mu \mathrm{M}$ \\
\hline $\begin{array}{l}\text { Dalton Trans., 2018, 47, } \\
7222\end{array}$ & $\begin{array}{l}\text { Triphenylamine- } \\
\text { functionalized luminescent } \\
\text { sensor }\end{array}$ & $72 \mathrm{nM}$ \\
\hline $\begin{array}{c}\text { J. Mater. Chem. C, 2016, } \\
4,11404\end{array}$ & $\begin{array}{l}\text { Cd (II)-organic } \\
\text { framework }\end{array}$ & $0.52 \operatorname{ppm}(3.75 \mu \mathrm{M})$ \\
\hline $\begin{array}{l}\text { Polym. Chem., 2018, 9, } \\
3832\end{array}$ & $\begin{array}{c}\text { 1,2,3-triazolyl based } \\
\text { conjugated microporous } \\
\text { polymer }\end{array}$ & $4.2 \mu \mathrm{M}$ \\
\hline $\begin{array}{l}\text { J. Hazard. Mater. 2018, 344, } \\
\text { 283-290 }\end{array}$ & $\begin{array}{l}\text { nitrogen-rich metal-organic } \\
\text { framework with }\end{array}$ & $0.17 \mu \mathrm{M}$ \\
\hline $\begin{array}{l}\text { Cryst. Growth Des. } 2018, \\
18,431-440\end{array}$ & Cd (II)-based MOF & $5 \mu \mathrm{M}$ \\
\hline \multirow{3}{*}{ This work } & NCD-1 (water medium) & $111.6 \mathrm{nM}$ \\
\hline & NCD-2 (water medium) & $68.9 \mathrm{nM}$ \\
\hline & NCD-2 (DMSO medium) & $36 \mathrm{nM}$ \\
\hline
\end{tabular}




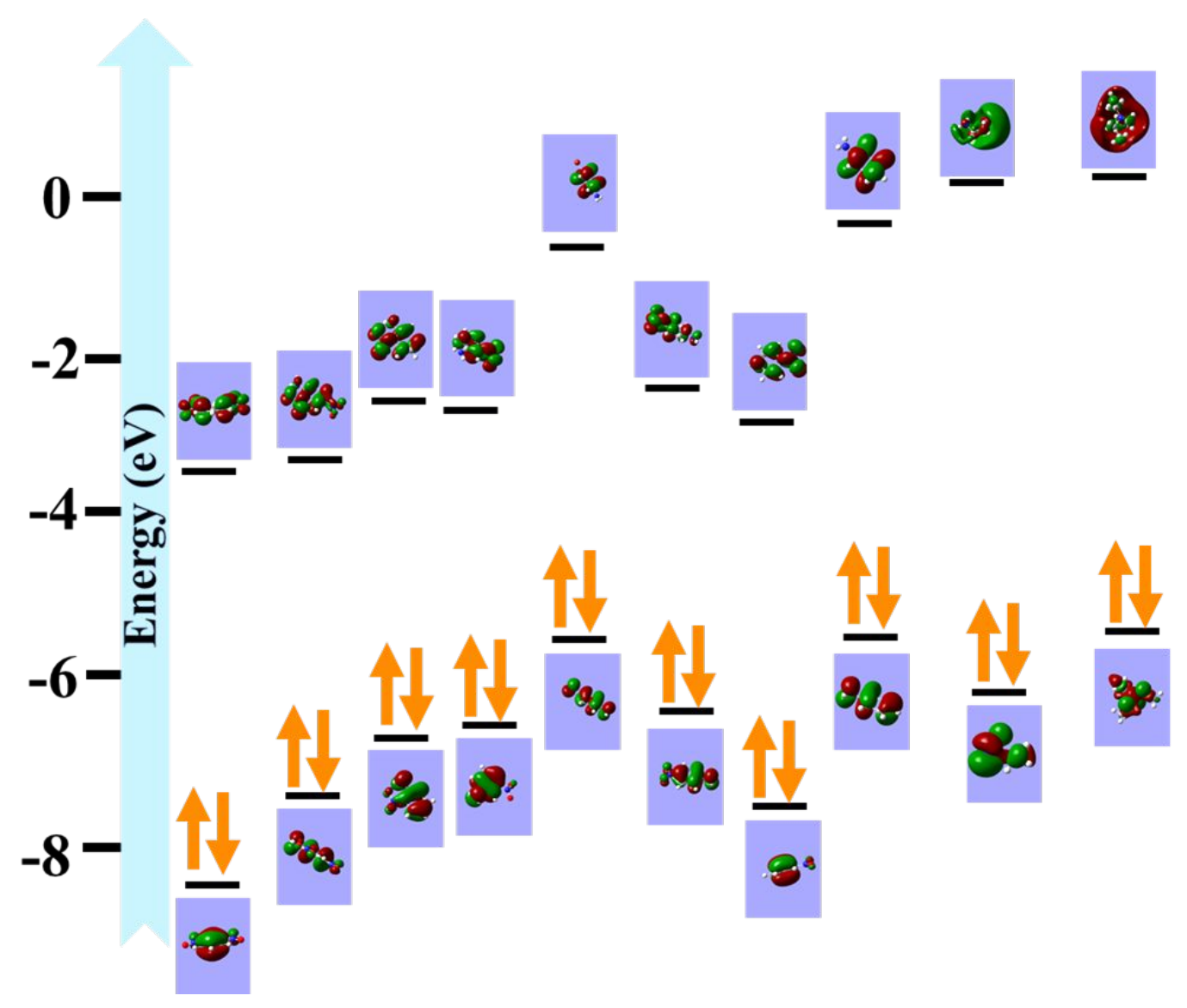

Figure S7. HOMO-LUMO energy diagram based on DFT analysis of nitro-compounds

\section{Calculation of FRET Efficiency}

$$
\text { FRET Efficiency }(E)=1-\frac{\tau_{D}-A}{\tau_{D}}
$$

Here, $\tau_{D}$ and $\tau_{D-A}$ denote the lifetimes of the nanoprobe NCDs in the presence and absence of 4-NA.

\section{Calculation of IFE}

$$
\frac{\mathrm{F}_{\text {corrected }}}{\mathrm{F}_{\text {observed }}}=\frac{2.3 \mathrm{dA} A_{\mathrm{ex}}}{1-10^{-\mathrm{dA} \mathrm{ex}_{\mathrm{ex}}}} 10^{\mathrm{gA} \mathrm{Am}_{\mathrm{em}}} \frac{2.3 \mathrm{sA} \mathrm{Am}_{\mathrm{em}}}{1-10^{-\mathrm{SA}} \mathrm{em}}
$$

Where, $\mathrm{F}_{\text {corrected }}$ is the corrected FL intensity after removing the IFE from the actual FL intensity; $F_{\text {observed }}$ is the actual FL intensity; $A_{e x}$ and $A_{e m}$ are the absorbance of NCDs at 360 $\mathrm{nm}$ and $450 \mathrm{~nm}$, respectively; $\mathrm{s}$ is the width of the excitation beam $(0.10 \mathrm{~cm})$; $\mathrm{g}$ is the distance between the edges of the cuvette and the excitation beam $(0.40 \mathrm{~cm}$ in this case $)$; $\mathrm{d}$ is the thickness of the cuvette $(1.00 \mathrm{~cm})^{1}$.

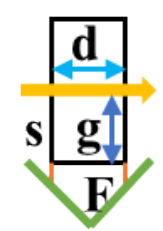




\section{Calculation of HOMO LUMO energies of NCDs}

Using the UV onset method, the calculated HOMO level is -5.12 and $-5.59 \mathrm{eV}$ for NCD-1 and $\mathrm{NCD}-2^{2-3}$.

band gap, $\mathrm{E}_{\mathrm{g}}(\mathrm{eV})=\frac{1240}{\mathrm{UV}_{\text {onset }}(\text { in } \mathrm{nm})}$

$\mathrm{E}_{\text {HOMO }}(\mathrm{eV})=-[\mathrm{E}$ (onset,ox vs $\left.\mathrm{Fc}+/ \mathrm{Fc})+4.8\right]$

$\mathrm{EFc} / \mathrm{Fc}+=0.09 \mathrm{eV}$

$\mathrm{E}_{\mathrm{LUMO}}=\mathrm{E}_{\mathrm{g}}+\mathrm{E}_{\mathrm{HOMO}}$

\begin{tabular}{|c|c|c|}
\hline Parameters & NCD-1 & NCD-2 \\
\hline Eg (eV) & 3.51 & 3.40 \\
\hline Oxidation potential & 0.88 & 0.41 \\
Eox (eV) & & -5.12 \\
\hline E $_{\text {HOMO }}(\mathrm{eV})$ & -5.59 & -1.72 \\
\hline $\mathrm{E}_{\text {LUMO }}(\mathrm{eV})$ & -2.08 & \\
\hline
\end{tabular}

Table S7. Decay curve fitting parameter of NCD-1 and after addition of $180 \mu \mathrm{M} 4-\mathrm{NA}$ in NCD-1 aqueous solution.

\begin{tabular}{|l|l|l|l|l|l|l|l|l|}
\hline Sample & $\mathbf{A}_{\mathbf{1}}$ & $\mathbf{A}_{\mathbf{2}}$ & $\mathbf{A}_{\mathbf{3}}$ & $\begin{array}{l}\boldsymbol{\tau}_{\mathbf{1}} \\
(\mathbf{n s})\end{array}$ & $\begin{array}{l}\boldsymbol{\tau}_{\mathbf{2}} \\
(\mathbf{n s})\end{array}$ & $\begin{array}{l}\boldsymbol{\tau}_{\mathbf{3}} \\
(\mathbf{n s})\end{array}$ & $\begin{array}{l}\boldsymbol{\tau}_{\text {avg }} \\
(\mathbf{n s})\end{array}$ & $\boldsymbol{\chi}^{\mathbf{2}}$ \\
\hline NCD-1 & 0.31 & 0.32 & 0.37 & 1.88 & 5.51 & 0.33 & 2.47 & 1.14 \\
\hline $\begin{array}{l}\text { NCD-1 } \\
+ \\
180 \mu \mathrm{M} \\
\text { 4-NA }\end{array}$ & 0.3 & 0.26 & 0.44 & 2.41 & 5.69 & 0.47 & 2.41 & 1.09 \\
\hline
\end{tabular}


Table S8. IFE parameter of NCD-1 and 4-NA system in aqueous solution.

\begin{tabular}{|c|c|c|c|c|c|c|}
\hline $\begin{array}{c}{[4-\mathbf{N A}]} \\
(\boldsymbol{\mu M})\end{array}$ & $\mathbf{A}_{\mathbf{e x}}$ & $\mathbf{A}_{\mathbf{e m}}$ & $\mathbf{C F}$ & $\mathbf{F}_{\text {observed }}$ & $\mathbf{F}_{\text {corrected }}$ & $\frac{\mathbf{F}_{\text {corrected }}}{\mathbf{F}_{\text {corrected }(\mathbf{0})}}$ \\
\hline 0 & 0.089842 & 0.00033 & 1.104892 & $2.17 \mathrm{E}+06$ & $2.40 \mathrm{E}+06$ & 1 \\
\hline 12 & 0.171027 & 0.044859 & 1.264502 & $1.89 \mathrm{E}+06$ & $2.39 \mathrm{E}+06$ & 1.002804 \\
\hline 24 & 0.255033 & 0.09121 & 1.449958 & $1.65 \mathrm{E}+06$ & $2.39 \mathrm{E}+06$ & 1.002792 \\
\hline 36 & 0.335467 & 0.135703 & 1.6484 & $1.43 \mathrm{E}+06$ & $2.35 \mathrm{E}+06$ & 1.018266 \\
\hline 48 & 0.415677 & 0.180366 & 1.868718 & $1.24 \mathrm{E}+06$ & $2.32 \mathrm{E}+06$ & 1.034723 \\
\hline 60 & 0.493502 & 0.223482 & 2.104627 & $1.07 \mathrm{E}+06$ & $2.26 \mathrm{E}+06$ & 1.060017 \\
\hline 75 & 0.609769 & 0.277292 & 2.474639 & 899454.4 & $2.23 \mathrm{E}+06$ & 1.07731 \\
\hline 90 & 0.686555 & 0.329602 & 2.793844 & 779317.2 & $2.18 \mathrm{E}+06$ & 1.101324 \\
\hline 105 & 0.779074 & 0.381666 & 3.187319 & 656353 & $2.09 \mathrm{E}+06$ & 1.146221 \\
\hline 120 & 0.870686 & 0.431589 & 3.613791 & 551078.1 & $1.99 \mathrm{E}+06$ & 1.20408 \\
\hline 135 & 0.961627 & 0.481057 & 4.080736 & 465142.9 & $1.90 \mathrm{E}+06$ & 1.263301 \\
\hline 150 & 1.046728 & 0.528266 & 4.564451 & 396277 & $1.81 \mathrm{E}+06$ & 1.325697 \\
\hline 165 & 1.133454 & 0.576349 & 5.103376 & 335407.6 & $1.71 \mathrm{E}+06$ & 1.400881 \\
\hline 180 & 1.218748 & 0.622775 & 5.676775 & 285981.5 & $1.62 \mathrm{E}+06$ & 1.477039 \\
\hline
\end{tabular}

Table S9. Fluorescence decay fitting parameters of NCD-2 and after addition of several concentrations of 4-NA in NCD-2 aqueous solution.

\begin{tabular}{|l|l|l|l|l|l|l|l|l|}
\hline Sample & $\mathbf{A}_{1}$ & $\mathbf{A}_{2}$ & $\mathbf{A}_{\mathbf{3}}$ & $\begin{array}{l}\boldsymbol{\tau}_{\mathbf{1}} \\
(\mathbf{n s})\end{array}$ & $\begin{array}{l}\boldsymbol{\tau}_{\mathbf{2}} \\
(\mathbf{n s})\end{array}$ & $\begin{array}{l}\boldsymbol{\tau}_{\mathbf{3}} \\
(\mathbf{n s})\end{array}$ & $\begin{array}{l}\boldsymbol{\tau}_{\text {avg }} \\
(\mathbf{n s})\end{array}$ & $\boldsymbol{\chi}^{\mathbf{2}}$ \\
\hline NCD-2 & 0.17 & 0.42 & 0.41 & 2.60 & 9.81 & 0.10 & 4.60 & 1.05 \\
\hline $\begin{array}{l}\text { NCD-2 + } \\
24 \mu \mathrm{M} 4-\mathrm{NA}\end{array}$ & 0.09 & 0.21 & 0.7 & 2.15 & 9.69 & 0.03 & 2.25 & 1.14 \\
\hline $\begin{array}{l}\text { NCD-2 + } \\
36 \mu \mathrm{M} 4-\mathrm{NA}\end{array}$ & 0.08 & 0.17 & 0.75 & 2.56 & 9.95 & 0.04 & 1.92 & 1.14 \\
\hline $\begin{array}{l}\text { NCD-2 + } \\
72 \mu \mathrm{M} 4-\mathrm{NA}\end{array}$ & 0.06 & 0.13 & 0.81 & 2.079 & 9.69 & 0.04 & 1.41 & 1.19 \\
\hline
\end{tabular}


Table S10. IFE parameter of NCD-2 and 4-NA system in aqueous solution.

\begin{tabular}{|c|c|c|c|c|c|c|}
\hline $\begin{array}{c}\text { [4-NA] } \\
(\boldsymbol{\mu M})\end{array}$ & $\mathbf{A}_{\mathbf{e x}}$ & $\mathbf{A}_{\mathbf{e m}}$ & $\mathbf{C F}$ & $\mathbf{F}_{\text {observed }}$ & $\mathbf{F}_{\text {corrected }}$ & $\frac{\mathbf{F}_{\text {corrected }}}{\mathbf{F}_{\text {corrected }(\mathbf{0})}}$ \\
\hline 0 & 0.0113 & 0.00277 & 1.013698 & 241724.5575 & 245035.7 & 1.000435 \\
\hline 12 & 0.02264 & 0.00319 & 1.027379 & 190408.5609 & 195621.7 & 1.253145 \\
\hline 24 & 0.03675 & 0.00594 & 1.04699 & 150796.5613 & 157882.6 & 1.552688 \\
\hline 36 & 0.05674 & 0.01036 & 1.075839 & 122510.9491 & 131802.1 & 1.859928 \\
\hline 48 & 0.06924 & 0.01206 & 1.092978 & 100146.7455 & 109458.2 & 2.239599 \\
\hline 60 & 0.08191 & 0.00985 & 1.106033 & 81530.55565 & 90175.51 & 2.718502 \\
\hline 72 & 0.09723 & 0.0097 & 1.124858 & 66466.96062 & 74765.87 & 3.2788 \\
\hline 90 & 0.11296 & 0.00965 & 1.144519 & 49706.84054 & 56890.45 & 4.309024 \\
\hline 102 & 0.12877 & 0.00987 & 1.164826 & 40862.45028 & 47597.64 & 5.150304 \\
\hline 120 & 0.14413 & 0.01118 & 1.186121 & 31084.91939 & 36870.47 & 6.648744 \\
\hline
\end{tabular}

Table S11. Decay fitting parameters of NCD-2 in DMSO solution and after addition of several concentration of 4-NA.

\begin{tabular}{|l|l|l|l|l|l|l|l|l|}
\hline Sample & $\mathbf{A}_{\mathbf{1}}$ & $\mathbf{A}_{\mathbf{2}}$ & $\mathbf{A}_{\mathbf{3}}$ & $\begin{array}{l}\boldsymbol{\tau}_{\mathbf{1}} \\
(\mathbf{n s})\end{array}$ & $\begin{array}{l}\boldsymbol{\tau}_{\mathbf{2}} \\
(\mathbf{n s})\end{array}$ & $\begin{array}{l}\boldsymbol{\tau}_{\mathbf{3}} \\
(\mathbf{n s})\end{array}$ & $\begin{array}{l}\boldsymbol{\tau}_{\text {avg }} \\
(\mathbf{n s})\end{array}$ & $\boldsymbol{\chi}^{\mathbf{2}}$ \\
\hline NCD-2 & 0.03 & 0.83 & 0.14 & 2.27 & 5.53 & 1.91 & 4.93 & 1.07 \\
\hline $\begin{array}{l}\text { NCD-2 } \\
+ \\
32 \mu \mathrm{M} 4-\mathrm{NA}\end{array}$ & 0.06 & 0.78 & 0.16 & 2.40 & 5.58 & 0.16 & 4.52 & 1.009 \\
\hline $\begin{array}{l}\text { NCD-2 } \\
+ \\
48 \mu \mathrm{M} 4-\mathrm{NA}\end{array}$ & 0.06 & 0.71 & 0.23 & 2.671 & 5.576 & 0.13 & 4.15 & 1.05 \\
\hline $\begin{array}{l}\text { NCD-2 } \\
+ \\
96 \mu \mathrm{M} 4-\mathrm{NA}\end{array}$ & 0.07 & 0.52 & 0.41 & 2.79 & 5.60 & 0.073 & 3.14 & 1.14 \\
\hline $\begin{array}{l}\text { NCD-2 } \\
+ \\
112 \mu \mathrm{M} 4-\mathrm{NA}\end{array}$ & 0.05 & 0.5 & 0.45 & 1.61 & 5.52 & 0.050 & 2.86 & 1.111 \\
\hline
\end{tabular}


Table S12. IFE parameter of NCD-2 and 4-NA system in DMSO solution.

\begin{tabular}{|c|c|c|c|c|c|c|}
\hline $\begin{array}{c}{[4-\mathbf{N A}]} \\
(\boldsymbol{\mu M})\end{array}$ & $\mathbf{A}_{\mathbf{e x}}$ & $\mathbf{A}_{\mathbf{e m}}$ & $\mathbf{C F}$ & $\mathbf{F}_{\text {observed }}$ & $\mathbf{F}_{\text {corrected }}$ & $\frac{\mathbf{F}_{\text {corrected }}}{\mathbf{F}_{\text {corrected }(\mathbf{0})}}$ \\
\hline 0 & 0.050933 & 0.004847 & 1.062729 & 1476520 & 1569141 & 1 \\
\hline 16 & 0.241759 & 0.0782 & 1.410895 & 1159870 & 1636455 & 1.042898 \\
\hline 32 & 0.428723 & 0.149617 & 1.833152 & 832423.1 & 1525958 & 0.97248 \\
\hline 48 & 0.617598 & 0.221001 & 2.350878 & 602263.3 & 1415847 & 0.902307 \\
\hline 64 & 0.803443 & 0.292618 & 2.96542 & 438969.2 & 1301728 & 0.82958 \\
\hline 80 & 0.991509 & 0.364819 & 3.70074 & 320364.5 & 1185586 & 0.755563 \\
\hline 96 & 1.170627 & 0.433579 & 4.517987 & 190776.9 & 861927.4 & 0.549299 \\
\hline 112 & 1.333301 & 0.497966 & 5.378456 & 141742.8 & 762357.4 & 0.485844 \\
\hline
\end{tabular}

Table S13. Real sample analysis for NCD-1.

\begin{tabular}{|l|l|l|l|l|l|}
\hline \multicolumn{1}{|c|}{ Sample } & \multicolumn{1}{|c|}{ Content } & \multicolumn{1}{c|}{$\begin{array}{c}\text { Added } \\
(\boldsymbol{\mu M})\end{array}$} & $\begin{array}{c}\text { Detected } \\
(\boldsymbol{\mu M})\end{array}$ & \multicolumn{1}{|c|}{ Recovery } & \multicolumn{1}{c|}{$\begin{array}{c}\text { RSD } \\
(\mathbf{n = 3 , \% )}\end{array}$} \\
\hline Lake water & Not found & 5 & 4.65 & 92.9 & 2.81 \\
\hline & & 10 & 9.66 & 96.6 & 4.56 \\
\hline Tap water & Not found & 5 & 5.85 & 117.1 & 5.46 \\
\hline & & 10 & 10.58 & 105.7 & 3.5 \\
\hline Lab waste water & 2.12 & 5 & 6.09 & 84.6 & 3.4 \\
\hline & & 10 & 10.23 & 83.7 & 5.28 \\
\hline
\end{tabular}

Table S14. Real sample Analysis for NCD-2

\begin{tabular}{|l|l|l|l|l|l|}
\hline Sample & Content & Added $(\boldsymbol{\mu M})$ & $\begin{array}{l}\text { Detected } \\
(\boldsymbol{\mu M})\end{array}$ & Recovery & $\begin{array}{l}\text { RSD } \\
(\mathbf{n = 3 , \% )}\end{array}$ \\
\hline Lake water & Not found & 4 & 4.59 & 114.8 & 2.61 \\
\hline Tap water & Not found & 4 & 8.86 & 110.8 & 2.68 \\
\hline & & 8 & 4.3 & 107.5 & 4.8 \\
\hline $\begin{array}{l}\text { Lab waste } \\
\text { water }\end{array}$ & 1.86 & 4 & 8.4 & 105 & 3.9 \\
\hline & & 8 & 5.16 & 84.5 & 1.13 \\
\hline
\end{tabular}




\section{Reference}

(1) Nandi, N.; Gaurav, S.; Sarkar, P.; Kumar, S.; Sahu, K. Multifunctional N-Doped Carbon Dots for Bimodal Detection of Bilirubin and Vitamin B12, Living Cell Imaging, and Fluorescent Ink. ACS Appl. Bio Mater. 2021, 4 (6), 5201-5211, DOI: 10.1021/acsabm.1c00371. (2) Thorat, K. G.; Kamble, P.; Ray, A. K.; Sekar, N. Novel pyrromethene dyes with N-ethyl carbazole at the meso position: a comprehensive photophysical, lasing, photostability and TDDFT study. Phys. Chem. Chem. Phys. 2015, 17 (26), 17221-17236, DOI: 10.1039/C5CP01741F.

(3) Yang, D.; Jiao, Y.; Huang, Y.; Zhuang, T.; Yang, L.; Lu, Z.; Pu, X.; Sasabe, H.; Kido, J. Two different donor subunits substituted unsymmetrical squaraines for solution-processed small molecule organic solar cells. Org. Electron. 2016, 32, 179-186, DOI: https://doi.org/10.1016/j.orgel.2016.02.009. 